\title{
Chance in the Everett interpretation
}

\author{
Simon Saunders
}

It is unanimously agreed that statistics depends somehow on probability. But, as to what probability is and how it is connected with statistics, there has seldom been such complete disagreement and breakdown of communication since the Tower of Babel (Savage [1954 p.2]).

\begin{abstract}
The notion of objective probability or chance, as a physical trait of the world, has proved elusive; the identification of chances with actual frequencies does not succeed. An adequate theory of chance should explain not only the connection of chance with statistics, but with degrees of belief, and more broadly the entire phenomenology of (seemingly) chance events and their measurement. Branching structure in the decoherencebased many worlds theory provides an account of what chance is that satisfies all these desiderata, including the requirement that chance involves uncertainty ${ }^{1}$
\end{abstract}

For present purposes I take Everettian quantum mechanics (EQM) to be the unitary formalism of quantum mechanics divested of any probability interpretation, but including decoherence theory and the analysis of the universal state in terms of emergent, quasiclassical histories, or branches, along with their branch amplitudes, all entering into a vast superposition. I shall not be concerned with arguments over whether the universal state does have this structure; those arguments are explored elsewhere (see e.g. Saunders et al [2010 Part 1,2]). (But I shall, later on, consider the mathematical expression of this idea of branches in EQM in more detail.)

My argument is that the branching structures in EQM, as quantified by branch amplitudes (and specifically as ratios of squared moduli of branch amplitudes) play the same roles that chances are supposed to play in one-world theories of physical probability. That is, in familiar theories, we know that

(i) Chance is measured by statistics, and perhaps, among observable quantities, only statistics, but only with high chance.

(ii) Chance is quantitatively linked to subjective degrees of belief, or credences: all else being equal, one who believes the chance of $E$ is $p$ will set his credence in $E$ equal to $p$ (the so-called 'principal principle').

(iii) Chance involves uncertainty; chance events, prior to their occurrence, are uncertain.

\footnotetext{
${ }^{1}$ This is a lightly edited reprint (with added footnotes and a new appendix) of 'Chance in the Everett interpretaiton', in Many Worlds? Everett, quantum theory and reality, S. Saunders, J. Barrett, A. Kent, and D. Wallace (Oxford 2010).
} 
Those seem the most important of the chance-roles.

My claim is that exactly similar statements can be shown to be true of branching in EQM. In the spirit of Saunders [2005] and Wallace [2006], we should conclude these branching structures just are chances, or physical probabilities. This is the programme of 'cautious functionalism', to use Wallace's term.

The argument continues: this identification is an instance of a general procedure in the physical sciences. Probabilities turn out to be functions of branch amplitudes in much the same way that colours turn out to be functions of electromagnetic frequencies and spectral reflectancies, and heat and temperature turn out to be functions of particle motions in classical statistical mechanics - and in much the same way that sensible perceptions (of the sort relevant to measurement and observation) turn out to be functions of neurobiological processes.

Just like these other examples of reduction, whether probability is thus explained, or explained away, it can no longer be viewed as fundamental. It can only have the status of the branching structure itself; it is 'emergent'. Chance, like quasiclassicality, is then an 'effective' concept, its meaning at the microscopic level entirely derivative on the establishment of correlations, natural or man-made, with macroscopic branching. That doesn't mean amplitudes in general (and other relations in the Hilbert space norm) have no place in the foundations of EQM - on the contrary, they are part of the fundamental ontology but their link to probability is indirect. It is simply a mistake, if this reduction is successful, to see quantum theory as at bottom a theory of probability.

\section{Explaining probability}

Functional reduction is not new to the sciences; functional reduction, specifically, of probability, is not new to philosophy. In any moderately serious form of physical realism a world is a system of actual events, arranged in a spatiotemporal array, defined in terms of objective, physical properties and relations alone. Where in all this are the physical probabilities? For physicalists, they can only be grounded on the actual structure of events - what Lewis has called a 'Humean tapestry' of events (Lewis [1986a xv-xvi]), whereupon the same identificatory project ensues as in EQM ${ }^{2}$. The links (i), (ii), (iii) stand in need of explanation whatever the physics, classical or quantum, one world or many. For the most part they have remained surprisingly hard to explain, even, and perhaps especially, in one-world theories ${ }^{3}$

\footnotetext{
${ }^{2}$ Lewis explicitly contemplated extending his 'tapestry of events' to quantum mechanics, acknowledging that novel quantum properties and relations (like amplitudes and relations among amplitudes) may have to be included; but only come the day that quantum mechanics is 'purified' (Lewis [1986a p.xi]) - 'of instrumental frivolity, of doublethinking deviant logic, and - most of all - of supernatural tales about the power of the observant mind to make things jump'. Quantum mechanics has certainly been purified in EQM.

${ }^{3}$ According to even so committed an empiricist as B. van Fraassen, a model of a probabilistic physical theory must include elements representing alternative possible sequences of outcomes, and the theory can be true 'only if alternative possible courses of events are real.' (van Fraassen
} 
But one of the three links, in the case of one-world theories, is easily explained - namely (iii), the link with uncertainty. Only allow that the dynamics is indeterministic (whether in a fundamental or effective sense), so that some kinds of events in the future are (at least effectively) unpredictable, then, for the denizens of that world, the future will in some respects at least be uncertain. This is how all the conventional theories of chance explain (iii) (with varying strengths of 'unpredictability'). But according to EQM all outcomes exist, and, we may as well suppose, are known to exist (we may suppose we know the relevant branching structure of the wave-function) - so the future is completely known. There is no uncertainty on this score.

This marks out EQM as facing a particular difficulty with (iii), the link with uncertainty, a difficulty encountered by none of the familiar theories of probability in physics. It has seemed so severe, in fact, that not only do the majority of critics of EQM believe that as a theory of probability it must rest on the links (i) with statistics and (ii) rationality alone (if it is to mean anything), but so do many of its friends ${ }^{4}$ The attempt to ground EQM on (i) and (ii) alone, disavowing all talk of probability or uncertainty, has been dubbed the fission programme. This article is intended as a counterbalance, and is skewed towards a defense of (iii), the link with uncertainty. As I shall argue in Section 3 and 4, the status of this link is not in fact so different in EQM than in conventional theories of chance, using a possible-worlds analysis, of a sort familiar to philosophers.

I have less to say on (i) and (ii), not because they are less important to the overall argument, but because arguments for them are either well-known (in the case of (i), the link with statistics) or given elsewhere in this volume (by Wallace, for (ii), the principal principle). Both links now appear to be in significantly better shape in EQM than in the other main theories of physical probability (classical statistical mechanics, deterministic hidden variable theories, and stochastic physical theories), none of which offer comparably detailed explanations of (i) and (ii). Instead they presuppose them.

First (i), the link with statistics. A first step was the demonstration that a largely formal condition of adequacy could be met: a quantum version of the Bernouilli ('law of large numbers') theorem could be derived 5 This says that in the limit of large numbers of trials, the probability of relative frequencies different from the predicted probabilities goes to zero. But this theorem follows from the rules of probability. Distinctive, in EQM, is that given an account of what chance processes /textitactually are (branchings), and given that in EQM we can model any measurement process as comprehensively as we please (including 'the observer' if need be) it becomes a purely dynamical question as to whether and how branch amplitudes can be measured. I will give some illustrative examples in Section 2 - but I take these arguments to be relatively uncontroversial.

Not so the arguments for (ii), the decision theory link. But even here, few would deny that there has been progress - and on two quite separate fronts,

[1980 p.197]).

${ }^{4}$ E.g. Papineau, [1996, 2010], Greaves [2004], [2007], Deutsch [1999, 2010].

${ }^{5}$ As first sketched by Everett in the 'long dissertation' (Everett [1973]). 
both involving the principal principle: the first (the sense already suggested) in showing why credence should track chance, as identified in our Everettian tapestry; the second in showing how we could have been led, by rational, empirical methods, to anything like that tapestry in the first place.

For the former argument, see Wallace [2010, 2012], deriving the principal principle 6 He shows that branching structures and the squared moduli of the amplitudes, insofar as they are known, ought to play the same decision theory role (ii) that chances play, insofar as they are known, in one-world theories. Whatever one might think of Wallace's axioms, as long as nothing underhand or sneaky is going on the result is already a milestone: nothing comparable has been achieved for any other physical theory of chance.

It is, however, limited to the context of agents who believe the world has the branching structure EQM says it has - it is a solution to the 'practical problem', the normative problem of how agents who believe they live in a branching universe ought to achieve their ends (without any prior assumption about probability). It has no direct role in confirming or disconfirming EQM as one of a number of rival physical theories by agents uncommitted as to its truth. It leaves the 'evidential problem' unsolved.

But on this front too there has been progress. Greaves [2007] and Greaves and Myrvold [2010] showed how confirmation theory (specifically Bayesian confirmation theory) can be generalized so as to apply to branching theories and non-branching theories evenhandedly, without empirical prejudice. The latter proved a representation theorem on the basis of axioms that are entirely independent of quantum mechanics. Nor, in keeping with the fission programme, do they make explicit or even tacit appeal to the notion of probability or uncertainty.

Wallace's axioms were likewise intended to apply even in the context of the fission programme. If acceptable that shows the argument for (ii) is independent of (iii). But if the argument for (iii) (with (i) and (ii)) goes through, then the present approach also promises a solution to the evidential problem. As an objection to the Everett interpretation, the problem only arises if it is granted that branching and branch amplitudes cannot be identified with chance and probabilities (which we take to mean are not in fact quantities that satisfy (i), (ii), (iii)), precisely the point here in contention. Show that they can, and the evidential problem simply dissolves. Or rather, since it can be read as a problem for every chance theory, it will have the same status in the Everett interpretation as it has in any other physical theory of chance, to all of which the Greaves and Myrvold analysis applies.

I have little more to say about (ii), the decision-theory link. What follows is

\footnotetext{
${ }^{6}$ Note added Sep 2016. This is the principal principle in the special instance of EQM. Wallace speaks instead of 'deriving the Born rule', but of couse there are many derivations of the Born rule (that have nothing to do with decision theory): of which Everett [1957] was the first. See e.g. Zurek [2005], for a derivation from locality, or Saunders [2004, 2005], for a derivation from operational and invariance principles respectively; Another notable [and greatly neglected] derivation was given by Lubkin [1979] (my thanks to Ted Jacobson for pointing this out to me.
} 
directed to (i), the link with statistics (Section 2), but mainly to (iii), the link with uncertainty 7

In Section 3 I show how branching in EQM is consistent with talk of uncertainty (following Saunders and Wallace [2008a]). That is arguably enough, with the results of Section 2 and in decision theory, to draw our main conclusion: branching in EQM should be identified with chancing, and mod-squared branch amplitudes with chance.

But there remains a contrary reading, according to which there is no place for uncertainty after all - or not of the right kind. Given sufficient knowledge of the wave-function, on this contrary reading uncertainty can at best concern present states of affairs, not future ones. In the final and more philosophical section I argue that the difference boils down to a choice of metaphysics, a choice that is under-determined by the mathematical structure of the theory. Since choices like this should be made to help in the understanding of a physical theory, rather than to frustrate it, the contrary reading should be rejected.

\section{Why chance is measured by statistics}

Here are a number of no-go facts about how physical probabilities are observed that we believe to be true, but that seem very difficult to explain on any conventional theory of physical probability:

(a) There is no probability meter that can measure single-case chance with chance equal to one.

(b) There is no probability meter that can measure chance on repeated trials with chance equal to one.

(c) There is no probability meter that can measure single-case chance with chance close to one.

(d) Absolute probability can never be measured, only relative probability.

(The list could be continued.)

On conventional thinking, someone who needs convincing of these facts about chance has not so much failed to understood a physical theory as the concept of probability. But if chance is a definite physical magnitude, it should (at least indirectly) be measurable, like anything else. Given the dynamics, and a theory complete enough to model the experimental process itself, these facts should be explained. And indeed no non-trivial function of the branch amplitudes can be measured in the ways mentioned in (a)-(d), according to EQM. This is a dynamical claim.

On the positive side, as to how (we know) chances are in practice measured:

\footnotetext{
${ }^{7}$ Note added Sep 2016. Of Wallace's axioms, 'branching indifference' (that branching per se, where the differences between branches do not matter, should be treated as irrelevant) has been thought particularly vulnerable. But if uncertainty is the right attitude in the face of branching, then I should be indifferent as to which of a number of persons is me, if they differ only in ways that do not matter to me. See Wilson [2011], [2013].
} 
(e) There are probability meters that measure chances on repeated trials (perhaps simultaneous trials) with chance close to one.

This is conventionally thought of as a consequence of the axioms of probability (the law of large numbers, or Bernouilli theorem) rather than of any physical theory; in turning it around, and deriving it rather from the dynamical equations of EQM, it identifies the appropriate physical quantities that are to count as probabilities instead.

There remains one other obvious fact without which one might well think no account of chance can so much as get off the ground: chance outcomes are typically mutually exclusive or incompatible - in which case only one outcome obtains (which one happens by chance). That is (where I explicitly add the qualification 'observable', as we are concerned with how chances can be measured):

Presupposition: Of two incompatible, observable, chance events, only one event happens

How can the presupposition be explained in EQM? In comparison with (a)(e) it seems to have the least to do with chance - the word 'chance' could be deleted from it entirely - but we surely do talk about chance in this way. It is presupposed by any application of the concept of chance that (at least sometimes) chances apply to incompatible outcomes. The concept of incompatibility enters at the the very beginning of any mathematical definition of a probability measure on a space of events (events are represented by sets, and inherit from set theory the structure of a Boolean algebra). And yet it seems to be violated straightforwardly in EQM, as Everett's crazy idea was that in a quantum measurement all outcomes happen.

If that was all there was to it it would be hard to understand why EQM was ever taken seriously by anyone. The answer is that the presupposition has two different readings, the one a physical or metaphysical claim - about what chances fundamentally are - and the other a claim about what is observable (by any observer), the phenomenology of chance.

On the first reading, the presupposition is straightforwardly denied; it is denied if the word 'chance' is deleted too. But we already knew this: this is simply a conflict between a many-worlds and a one-world theory. Likewise for the presupposition as a metaphysical claim: we already knew EQM challenges a number of a priori claims. It is only as an epistemological claim - as to the observed phenomenology - that the presupposition had better still make sense. But so it does: EQM explains it very simply. No two incompatible chance outcomes can happen in the same branch. And since the apparatus, and the observer, and the entire observable universe, are branch-relative - they are 'in-branch' structures - no observer can ever witness incompatible outcomes simultaneously 8 In this

\footnotetext{
${ }^{8}$ If this is thought to be question-begging: there does not exist a perception of two incompatible outcomes, according to EQM, treating perception biochemically, or indeed as any kind of record-making process. (This point goes back to Everett [1957] and, ultimately, the von Neumann model of measurement. See also Gell-Mann and Hartle [1990] and Saunders [1994].)
} 
second sense, then, the presupposition is rather elegantly dealt with in EQM 'elegantly', because it follows directly from its account of what a chance set-up is, and from what chance is (one should not delete the word 'chance'!).

The other everyday ('no-go') facts about probabilities follow, not from branching alone, but from the unitarity of the equations of motion. Because I do not think this claim is particularly controversial I will simply illustrate it with a proof of (a), for relative probabilities (meaning a quantity that concerns the relationships between chancy outcomes). Consider then a microscopic system in the state

$$
\left|\varphi_{c}\right\rangle=c\left|\varphi_{+}\right\rangle+\sqrt{1-|c|^{2}}\left|\varphi_{-}\right\rangle .
$$

For simplicity, model the measurement process as a 1-step history, using the Schrödinger picture, with initial state $|\omega\rangle \otimes\left|\varphi_{c}\right\rangle$ at $t=0$ where $|\omega\rangle$ is the state of the rest of the universe. Let the configuration describing (among other things) the apparatus registering (a function of) the amplitude $c$ at time $t$ be $\alpha(c)$. Let the projection onto this configuration be $P_{\alpha(c)}$ and let the associated Heisenberg-picture projection be

$$
P_{\alpha(c)}(t)=e^{i H t / \hbar} P_{\alpha(c)} e^{-i H t / \hbar}
$$

(See appendix 1 for how quantum histories are defined as time-ordered products of such projectors.) The no-go fact we need to establish is that there is no unitary dynamics $U_{t}=e^{-i H t / \hbar}$ by means of which:

$$
|\omega\rangle \otimes\left|\varphi_{c}\right\rangle \rightarrow U_{t}|\omega\rangle \otimes\left|\varphi_{c}\right\rangle=U_{t} P_{\alpha(c)}(t)|\omega\rangle \otimes\left|\varphi_{c}\right\rangle
$$

for variable $c$. If there were it would also follow that for $c^{\prime} \neq c$ :

$$
|\omega\rangle \otimes\left|\varphi_{c^{\prime}}\right\rangle \rightarrow U_{t} P_{\alpha\left(c^{\prime}\right)}(t)|\omega\rangle \otimes\left|\varphi_{c^{\prime}}\right\rangle .
$$

But the inner product of the LHS of the two initial states can be as close to one as desired, whilst that of the vectors on the RHS must be zero, as $\alpha(c)$ and $\alpha\left(c^{\prime}\right)$ must differ macroscopically for sufficiently large $\left|c-c^{\prime}\right|$, a contradiction. Thus not even relative probabilities can be measured deterministically. (The argument for absolute probabilities is even simpler, and depends only on the linearity of the Schrödinger equation.)

Now for (e), how (relative) chances can be measured according to EQM, with high chance. Take a number $N$ of subsystems, each (to a good approximation) in state $\varphi_{c}=c \varphi_{+}+\sqrt{1-|c|^{2}} \varphi_{-}$, and arrange the linear dynamics so that

$$
\left|\omega \otimes \varphi_{ \pm}\right\rangle \rightarrow U_{t} P_{\alpha( \pm)}(t)\left|\omega \otimes \varphi_{ \pm}\right\rangle
$$

i.e. the von Neumann model of measurement, where $\alpha(+)$ is a configuration in which the apparatus reads 'spin up' and $\alpha(-)$ 'spin down'. Applied to an initial state of the form $|\omega\rangle \otimes\left|\varphi_{c}\right\rangle$, it yields the superposition

$$
|\omega\rangle \otimes\left|\varphi_{c}\right\rangle \rightarrow U_{t} P_{\alpha(+)}(t)\left|\omega \otimes \varphi_{c}\right\rangle+U_{t} P_{\alpha(-)}(t)\left|\omega \otimes \varphi_{c}\right\rangle
$$


in which the first vector has norm $|c|$ and the second has norm $\sqrt{1-|c|^{2}}$. That is, the amplitudes $c, \sqrt{1-|c|^{2}}$ of components in a microscopic superposition have been promoted to macroscopic branch amplitudes. Consider now repeated measurements of $N$ microscopic systems all in the same state $\left|\varphi_{c}\right\rangle$, whether sequentially repeated in time, or measured all at once. The latter is the simplest to model, assuming the $N$ apparatuses are non-interacting: the result at time $t$ will be a superposition of vectors at $t$ of the form

$$
\left|\alpha_{f}(t)\right\rangle=U_{t} P_{f(1)}(t) \otimes \ldots \otimes P_{f(N)}(t)|\omega\rangle \otimes\left|\varphi_{c}\right\rangle \otimes \ldots \otimes\left|\varphi_{c}\right\rangle
$$

where $f(k), k=1, \ldots, N$ is either +1 or -1 . Those with the same relative frequencies $M / N$ will all have the same norm, i.e.:

$$
\left.|| \alpha_{f}, t\right\rangle\left.|=| c\right|^{M}{\sqrt{\left(1-|c|^{2}\right)}}^{N-M} ; \sum_{k=1}^{N} f(k)=2 M-N .
$$

The unitary evolution to time $t$ is:

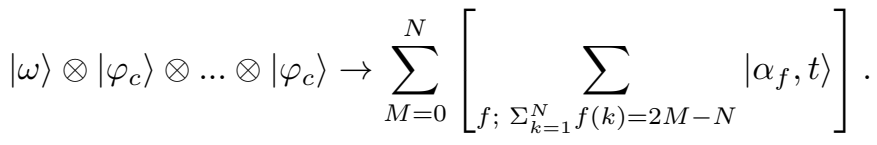

The right-most summation, for fixed $M, N$, is over all $N ! / M !(N-M)$ ! distinct $f$ 's, all with the same norm Eq.(2). Since $\left\langle\alpha_{f}, t \mid \alpha_{g}, t\right\rangle=0$ for $f \neq g 9^{9}$ the squared norm of the RHS of Eq.(3) is

$$
\left.\sum_{M=0}^{N} \frac{N !}{M !(N-M) !}|| \alpha_{f}, t\right\rangle\left.\right|^{2}=\sum_{M=0}^{N} x(M)
$$

where $x(M)$ is:

$$
x(M)=|c|^{2 M}\left(1-|c|^{2}\right)^{N-M} \frac{N !}{M !(N-M) !} .
$$

For $N$ large, this function is strongly peaked about $M / N=|c|^{2}$. The sum of norms of vectors with the 'right' relative frequency is much larger than the sum of norms of vectors with the 'wrong' relative frequency. In this sense measured relative frequencies close to $|c|^{2}$ are found on relatively high amplitude branches.

It was rather crucial to this argument that we consider only relative frequency - we don't care about precisely which particles were recorded as 'spinup' and 'spin-down' (or which of our $N$-apparatuses recorded which result). That is, our measurement protocol requires that the measurements be treated as 'exchangeable', in de Finetti's sense (Greaves and Myrvold [2010 pp.277-80]). This is an axiom of de Finetti's approach, whereas in EQM it is explained.

\footnotetext{
${ }^{9}$ For sequences of measurements in time, the consistency condition is needed at this point: see appendix 1.
} 
Might some other method be found by which functions of amplitudes may be measured, flouting (a)-(d)? Perhaps ${ }^{10}$ but that is unlikely to make for objection if, like EQM, it is based on the unitary formalism of quantum mechanics. If based on a rival theory, which is empirically successful, that will anyway spell the end of quantum mechanics (and with it EQM).

Now suppose, fancifully if you will, that we are entitled to identify chances with functions of branch amplitudes. Then in summary we have just shown: chances will generally be associated with incompatible observable outcomes, only one of which can happen at each time; they may not be measured in a way that is not itself chancy, and single-case chance cannot be measured at all. They can only be measured by running a chance (branching) process repeatedly, or by a single trial involving a large number of similarly prepared systems, and listing the relative frequencies (taking care to neglect the order of outcomes, or which system had which outcome). The measurement will be veridical, however, only with high chance, given the number of systems involved is sufficiently large. None of these facts can be explained by any conventional physical theory of probability (rather, they are presupposed).

\section{Why chance involves uncertainty}

We cannot, however, identify chance set-ups with branch set-ups, and chances with functions of amplitudes, failing an account of (iii), the link with uncertainty. In the absence of this, to arrive, by the Deutsch-Wallace representation theorem, at an agent's credence function (in conformity with the Born rule), raises a puzzle on its own. For what does a rational agent believe to some degree when she uses that credence function? Given the theorem, certainly we can explain credence by reference to behaviour; but in this case, better, perhaps, to simply explain it away. A rational agent must order her priorities somehow, whether or not there is anything of which she is uncertain; but it is hard to see how is it possible to have degrees of belief different from one and zero if you know everything there is to know.

The functionalists' response is to more or less recapitulate the representation theorem: credences derive their meaning from their function in rational action, in determining expected utilities, and hence agents' preferences. They will also note that meaning is determined by use - that if anything has been agreed by philosophers of language in recent decades it is:

Words have no function save as they play a role in sentences: their semantic features are abstracted from the semantic features of sentences, just as the semantic features of sentences are abstracted from their part in helping people achieve goals or realize intentions. (Davidson [2001, p. 220])

\footnotetext{
${ }^{10}$ For example, 'protective measurements' (Aharonov and Vaidman [1993]), although see the criticism of Uffink [2000]); or 'weak measurements' (Aharonov et al [1988]).
} 
So there is every reason to talk of expected utilities and credences. By the same broadly functionalist philosophy, if these credences play the same role that credences about chance events play, they are credences about chance events. Talk of uncertainty and unpredictability then falls into place along with the rest of our ordinary use of words.

I think this argument is essentially correct, but it leaves unanswered - brief explanations please! - just how talk of uncertainty is to fall into place, and just what to say in answer to the question of what these degrees of belief are about.

First a warm-up. In the previous section I argued that functions of branch amplitudes are measured - they are manifested in-branch - in just the way that chances are measured. A part of that argument was that the presupposition about incompatible outcomes follows (rather straightforwardly) from EQM: on each trial, since macroscopically distinct, only one outcome can be obtained in each branch. So from the point of view of accumulating information that can actually be used - 'in-branch' information - events are registered sequentially, just as they are in a non-branching theory. This was what Everett [1957] took pains to show. But isn't sequential increase in information increase in knowledge? So isn't there something that is being learned in each branch?

Consider a concrete example. Alice, we suppose, is about to perform a SternGerlach experiment. She understands the structure of the apparatus and the state preparation device, and she is convinced EQM is true. In what sense does she learn, post-branching, something new? The answer is that each Alice, postbranching, learns something new (or is in a position to learn something new) each will say something (namely, 'I see the outcome is spin-up (respectively, spindown), and not spin-down (respectively, spin-up)') that Alice prior to branching cannot say. It is true that Alice, prior to branching knows that this is what each successor will say - but still she herself cannot speak in this way.

To make this vivid, imagine that each of her successors simply closes her eyes, when the result is obtained; in that state, each is genuinely ignorant of the result (so-called 'Vaidman ignorance'; Vaidman [1998]). This has usually been considered (by Vaidman himself) as ignorance that Alice, prior to branching, does not have, but it can be turned around the other way: each Alice, closing her eyes, perpetuates a state of ignorance that she already had.

The implication of this line of thought ${ }^{11}$ is that appearances notwithstanding, prior to branching Alice does not know everything there is to know. What is it she does not know? I say 'appearances notwithstanding', for of course in one sense (we may suppose) Alice does know everything there is to know; she knows (we might as well assume) the entire corpus of impersonal, scientific knowledge. But what that does not tell her is just which person she is - or where she is located - in the wave-function of the universe.

The point is a familiar one to philosophers. One can know that there are such-and-such people, but not which one of them is me. Or that such-and-such events occur at various places, but not which of those places is here; or times, but not which of those times is now.

\footnotetext{
${ }^{11}$ Picked up also by Ismael [2003], although she develops it in a rather different way.
} 
Such knowledge that is omitted is sometimes called 'indexical' knowledge, by philosophers, also 'knowledge de se', and 'self-locating knowledge' (we shall use the latter). But why should knowledge like this be lacking? Vaidman has Alice hide her eyes. Another example, due to J. R. Perry, likewise suggests some kind of impairment is needed. Perry asks us to consider an amnesiac who has lost his way in the library at Stanford. He does not know who he is; he does not know where he is - not even were he to read every book in the library, not even if he were to read his own biography, would he be any the wiser. But Alice is not so impaired. She does not hide her eyes, and no more is she an amnesiac. She sees where she is in the wave-function of the universe and self-locates accordingly and differently - from where her successors locate themselves, for, obviously, she is at a different place from them. She is not ignorant of anything her successors know; she simply reports different self-locating facts from them.

And so she would, but only given certain assumptions as to what, exactly, she is - of how she is represented in the physics. For example, if she at $t_{j}$ is represented by the configuration $\beta_{j}$, or by the Heisenberg-picture vector $\left|\beta_{j}\right\rangle=$ $P_{\beta_{j}}\left(t_{j}\right)|\Omega\rangle$, where $|\Omega\rangle$ is the universal state, with her successors at $t_{k}>t_{j}$ similarly represented by configurations $\beta_{k}, \beta_{k}^{\prime}$, or vectors $\left|\beta_{k}\right\rangle,\left|\beta_{k}^{\prime}\right\rangle$, the result surely follows. But this treats her as strictly a momentary thing, independent of the history in which she is located. There is an alternative: if she at $t_{j}$ is represented instead by an entire history $\beta$, indexed by $t_{j}$, then of course, at $t_{j}$, she does not know which history or branch vector is hers, and quite the opposite result follows.

Which of the two is correct? But questions like these (or rather their classical counterparts) are well known in metaphysics (and specifically personal identity). Most philosophers are agreed they cannot be settled on the basis of the physics alone. Here then is a metaphysics friendly to the Everett interpretation: let persons be spacetime worldtubes from cradle to grave (in the jargon, 'maximal continuants'). If Alice is a person (of course she is) then we must say, even prior to branching at $t_{j}$, that there are many Alice's present, atom-for-atom duplicates up to $t_{j}$, each behaving in exactly the same way and saying just the same words. If that is the right metaphysical picture for EQM, then Alice should be uncertain after all - each Alice should be uncertain - for each as of $t_{j}$ does not (and as a matter of principle, cannot) know which of these branching persons is she.

That, I take it, establishes that the question of whether or not there is uncertainty in the Everett interpretation can be settled either way, depending on a metaphysics of personal identity. But rather than invoke a metaphysical assumption, we can make do with a different kind of claim - a proposal, not about the ultimate natures of persons, but about the reference of the word 'person' in EQM terms. The proposal is that talk of persons (and things) be relativized as to branch 12

S1 By a 'person' or 'thing' is meant a branch-part or ordered pair $(\beta,|\alpha\rangle)$, where

\footnotetext{
${ }^{12}$ This semantics was discussed briefly in Wallace [2005], [2006] and in more detail in Saunders and Wallace [2008a], on which the argument that follows is based.
} 
$\alpha \in \beta$.

Here ' $\alpha \in \beta$ ' means the sequence of configurations $\beta$ is obtained by a coarsegraining of $\alpha$, which we suppose is temporally and spatially much more finely grained. Likewise 'a person at time $t_{j}$ ' is represented by an ordered triple $\left(\beta_{j}, \beta,|\alpha\rangle\right)$ (if a person is an entire history), where $\alpha \in \beta \in \beta_{j}$, or by an ordered pair $\left(\beta_{j},|\alpha\rangle\right)$ (if a person is a stage). More is needed for tensed sentences (involving 'was', 'is', 'will be' etc.), but for this, and on the question of how S1 can be justified, see Section 4.

For now note $S 1$ 's virtues. First, it is clearly permissible; it makes use of nothing but the available mathematics of EQM in terms of branch vectors $|\alpha\rangle$ and sequences of configurations $\alpha, \beta$ etc., as ordered pairs $\{(\beta,|\alpha\rangle) ; \alpha \in \beta\}{ }^{13}$. True, this requires the consistent histories formalism, in which branch vectors are Heisenberg-picture vectors (so that a branch vector $|\alpha\rangle$ describes the entire history $\alpha$, not any particular instant of it). The perspective is atemporal. But branching itself is a process defined by the dynamics over time: the idea of a decoherence basis, defined at an instant of time independent of what comes before and what comes after, is a fiction.

Second, using $S 1$, not only is Alice entitled - bound - to be unsure of the outcome of the experiment, but she has a genuine gain in knowledge when it is learned. For after branching, using S1, on observing the outcome, each Alice self-locates better than she did before - knows more than she did before - and so has learned something that, prior to branching, she could not have known. Vaidman's ignorance is ignorance each Alice already had. Likewise, there can be no algorithm, whose input is data about a branch up to one time, and whose output uniquely specifies that branch at a later time; for the same algorithm must operate in every other branch which is exactly the same up to that time but which differs thereafter. Branching events are algorithmically uncertain too - they are indeterministic.

Third, $S 1$ is neutral on some (but not all) metaphysical questions about personal identity. Specifically, those that arise given 4-dimensionalism, assuming a single world, are likely to play out the same under $S 1$, in the context of branching worlds, if only it is permitted to relativize one's favoured candidate for $\beta$ to worlds (represented by Heisenberg-picture branch vectors $|\alpha\rangle$, where $\alpha \in \beta)$.

And the bottom line: under $S 1$, prior to branching, uncertainty is assured: Alice doesn't know if she will see spin-down or spin-up, as she doesn't know which branch she is in. Unless some hidden contradiction is involved, $S 1$ is the right rule for making sense of quantum mechanics in realist terms.

Conclusion. Branching, the development of superpositions of the universal state with respect to the decoherence basis, plays all the chance roles (i), (ii), (iii): it produces the same phenomenology as chance, the squared norms of branch amplitudes are of practical relevance to decision theory in the way that chances normally are, and using SI, branching involves uncertainty, just

\footnotetext{
${ }^{13}$ Contrary to Kent [2010 p.346].
} 
as chances do. In EQM, branching and squared norms of branch amplitudes are demonstrably functionally equivalent to chance, in these three central respects; therefore they are chance processes, and chances are these physical magnitudes.

\section{Overlap and divergence}

There are alternatives to the rule $S 1$, however. Invoking it seems to compromise a chief selling point of the Everett interpretation, which is that many worlds follows from the unitary dynamics, with no added principles or special assumptions. That is what puts the Everett interpretation in a class of its own when it comes to the quantum realism problem: there are plenty of avenues for obtaining (at least non-relativistic) one-world theories if we are prepared to violate this precept.

On the other hand $S 1$ is on the face of it just a semantic rule - it is merely a linguistic matter. The referents of terms are constrained by their contexts of use, granted; but over and above those constraints, not even in the God's eye view is their meaning determined. It is up to us to say what, precisely, among the structures in the universal state, our words really mean. And if that is all there is to uncertainty, nothing much should hang on the matter either way. The challenge remains: to justify the probability interpretation of EQM on the grounds of (i) and (ii) alone.

I think there is something right about this argument. Whether or not there is genuine uncertainty in the Everett interpretation appears rather less substantive than might have been thought. But in that case, let satisfaction of the chance roles (i) and (ii) be enough to count for branching to count as chance; our conclusion stands 14

But on three counts this would be too quick. The first is that for many, $S 1$ really is a metaphysical claim, for all my talk of semantics - and that there is a substantive question of whether it is true. Certainly there are alternative metaphysical claims that could be made that cause problems to $S 1$. I shall have something to say about this sort of argument, but not much: for I do not believe there are metaphysical truths of this sort, independent of ordinary language and natural science. Or let me put the point more constructively: metaphysics should primarily be answerable to language and to science; it should be 'naturalized' (Saunders [1997], Ladyman and Ross [2007]). If this is right $S 1$ may still be a metaphysical claim, a claim of substance, but in the naturalized sense.

The second count is that we should take rather more seriously the task of accounting for language use, for that too is emergent structure, a part of the physical world to be studied as such by scientific means. As with any form of functional reduction, we know what we are looking for in advance: the problem is to identify the right sort of structure at the more fundamental physical level to account for phenomena that we are already familiar with at the less. Devising

\footnotetext{
${ }^{14}$ This is not the fission programme, which would have us renounce talk of objective chance and genuine probability (along with uncertainty).
} 
semantic rules, whose truth conditions are fixed by reference to the underlying theory, is only a variation on the same procedure - it is reductionism as it is appropriate to linguistics. It is no different in kind from fixing on certain variables, for example hydrodynamical variables, in decoherence theory, to derive quasiclassicality. Sure, there remain the wrong variables, ones that give no hint of classicality; and wrong semantic rules too, which are inadequate to explaining our linguistic behaviour - and which give no hint of uncertainty. They should be eschewed.

This argument is a variant of Wallace's [2005], which puts the matter in terms of 'the principle of charity'. This principle says that the most important criterion of 'good' translation, in the radical case, where no prior standard of translation has been established, is that it maximizes truth. Working out what to say about branching, if EQM is true, is like radical translation - of how to translate into our own tongue the expressions of some alien language on the basis of observable linguistic behaviour alone. If EQM is true, it has always been true, and we have always used words like 'uncertainty' and 'chance' in the context of branching so - like it or not! - that is what those words have always been about. Whatever we might say about novel scenarios like teleportation machines or brain transplants (the ways in which philosophers have tended to imagine persons being divided) our use of words in ordinary contexts is not in doubt. The referents of ordinary words and truth conditions of everyday sentences (as specified in a physical theory) may wait to be correctly identified, but the criteria for 'correctness' is decided, not by metaphysics, but by their adequacy, as follows from the theory, to make sense of what we ordinarily say.

Greaves herself accepts this argument (Greaves [2007 p.124]), but not all Everettians do, and surely not all skeptics of EQM do. But this dispute is independent of EQM per se. It should be settled if possible by some other example of the general method - say, in the arena of a semantics for temporal affairs, involving tense and becoming, in classical spacetime theories (as argued by Saunders [1996], Wallace [2005]), or in the arena of a semantics for agency, moral responsibility, and free-will, in deterministic theories.

The third count on which the case for uncertainty can be too quickly deflated is a strengthened version of the first. I have already hinted at it: maybe $S 1$ does harbour some hidden contradiction, or some failure of integrity more broadly construed. The semantics should not misrepresent our situation, were EQM true.

This, it seems to me, is the only serious concern, and the only one I shall consider in the rest of this chapter. But it needs explaining. The very worry seems strange. How can a sentence misrepresent a theory, if it is true by that very theory?

Some more detail on the semantics will be helpfu 15 . Let $\alpha=\left\langle\alpha_{+}, . ., \alpha_{-}\right\rangle$, where $t_{+}$is much later than any time we are interested in, and $t_{-}$is much earlier. Call branches like this maximal. By 'world' I mean maximal branches,

\footnotetext{
${ }^{15}$ Other rules may be possible as well: see Wallace [2006] for some alternatives. The rules that follow are illustrative.
} 
represented by maximal branch vectors. Let $F$ be true of some branches, false of others. Then since, by $S 1$, speakers and things are parts of branches, so are utterances:

S2 An utterance of ' $F$ ' in branch $|\alpha\rangle$ is true if and only if $F$ is true in $\alpha$.

Now for tensed statements. Let $\alpha^{+}(t) \underset{\text { def }}{=}\left\langle\alpha_{+}, . ., \alpha_{k}\right\rangle \ni \alpha, t_{k}>t \geq t_{k-1}$ be the future of $\alpha$ at $t$; let $\alpha^{-}(t) \underset{d e f}{=}\left\langle\alpha_{k}, . ., \alpha_{-}\right\rangle \ni \alpha, t_{k+1}>t \geq t_{k}$ be the past of $\alpha$ at $t$. Let $F$ be true at some times, false at others (it is an 'occasion sentence', in Quinean terms). The rule for future physical contingencies is:

S3 An utterance of ' $F$ will be the case' in branch $|\alpha\rangle$ at $t$ is true if and only if $F$ is true in the future of $\alpha$ at $t$.

A first stab at a rule for future possibilities is:

S4 An utterance of ' $F$ might happen' in branch $|\alpha\rangle$ at $t$ is true if and only if for some branch $\left|\alpha^{\prime}\right\rangle, F$ is true in the future of $\alpha^{\prime}$ at $t$, where the past of $\alpha^{\prime}$ and $\alpha$ at $t$ is the same.

But the latter will obviously need to be restricted to branches vectors $\left|\alpha^{\prime}\right\rangle$ whose norm, conditional on $\alpha^{-}(t)$, is non-negligible (or else it will turn out that pretty well anything might happen). And similarly for counterfactuals.

$S 2$ and $S 3$, fairly obviously, satisfy the principle of charity. $S 4$ promises to, at least if 'might happen' is taken to mean 'might happen by chance' - that is, in accordance with the laws of quantum mechanics. Apart from this proviso, it fits well enough with standard ideas in modal metaphysics. One has only to replace 'branch $|\alpha\rangle$ ' by 'possible world' and the 'sameness of the past' relation entering in $S 4$ by a 'nearest counterpart' relation (Lewis [1973]) - although philosophers typically are interested in a notion of possibility of much broader scope.

Now notice that the 'sameness of the past' relation is neutral on the question of whether, in the past, (maximal) branches (or rather spatiotemporal parts of such branches) are numerically the same, or only qualitatively the same. They are neutral on the question of whether (to use a technical term in philosophy) they 'overlap' or merely 'diverge'. But this difference is crucial, say metaphysicians. Rules like $S 1-S 4$ have been offered by philosophers (almost always) as a semantics for the latter sort, for 'diverging' worlds, not for the former - or, as philosophers also call overlapping worlds, 'branching' worlds.

Which is it, in EQM? This question is in danger of being settled on the basis of an accident of terminology. Let us agree that 'branching' means the development of superpositions with respect to the decoherence basis. For the sense intended by philosophers - where there are numerical identities among spatiotemporal parts - we will speak of 'overlap'. Mundane examples of overlap are everywhere. Thus roads overlap if they share the same stretch of asphalt; houses and roofs, cars and steering wheels, hands and fingers, all overlap. Overlapping, like Everettian branching, supposedly has a formal definition too, but 
not in terms of Hilbert space structure. Rather, it is defined in terms of 'mereology', the general theory of parts and wholes. (I shall come back to this shortly.)

As for 'divergence', its definition is more equivocal. It is sometimes used to mean that there exist no physical relations between worlds, contrary to the situation for worlds in EQM ${ }^{16}$ but we shall take it as simply the opposite of overlap: 'diverging' means 'non-overlapping'.

Now to the point: if worlds overlap in EQM then (definitionally) there are genuine transworld identities. The very same thing exists in different worlds. In particular, since branching is massive in EQM, if worlds overlap then the very same person is part of vast numbers of worlds, differing, perhaps, only in respects remote in space and time - differing, maybe, only after centuries. All this is contrary to $S 1$. In that case any uncertainty to be had can have nothing to do with a metaphysics of personal identity. But is there any uncertainty to be had?

The worry is not that overlapping worlds are unintelligible or inconsistent; it is that they make nonsense of ordinary beliefs. As Lewis put it 17

Respect for common sense gives us reason to reject any theory that says that we ourselves are involved in branching [overlapping].... But we needn't reject the very possibility that a world branches [overlaps]. The unfortunate inhabitants of such a world, if they think of 'the future' as we do, are of course sorely deceived, and their peculiar circumstances do make nonsense of how they ordinarily think. But that is their problem; not ours, as it would be if the worlds generally branched [overlapped] rather than diverging. (Lewis [1986b p.209].)

Diverging worlds, composed of objects and events that do not overlap (that are qualitatively but not numerically identical) do not suffer from this problem.

Of course 'common sense' does not cut it much in the physical sciences; and Lewis' final sentence could not more comprehensively beg the question. But let us grant this much: he who believes he is contained in each of a number of worlds cannot also wonder which of them he's in.

We are at the nub of the matter: do worlds - maximal branches, sequences of relative configurations of particles and fields, as described by EQM - overlap in the philosophers' sense, or do they diverge?

We should discount two considerations. First, the coincidence in the terminology 'branching' (because as introduced by Everett, it referred to the mathematical formalism of quantum mechanics, not to the philosophers' criterion of overlap). Second, the fact that worlds in EQM do not diverge in the sense

\footnotetext{
${ }^{16}$ This can cause some confusion, evident in Saunders [1998 Sec.5], where I erroneously said that 'fatalism' (a near-neighbour to the position I am currently defending) involved the replacement of the superposition of histories (the universal state) by an incoherent mixture of histories, and must thus be rejected.

${ }^{17}$ Not everyone agrees with Lewis on this point. Thus Johnston [1989] suggested the rule $S 3$ in the explicit context of overlapping persons, arguing that semantic rules like this were underdetermined by the metaphysics.
} 
of being physically disconnected (they are not physically disconnected, because they superpose, but the issue is whether or not they overlap).

Their remains another consideration, however. There is a clear parallel with simultaneous rather than temporal overlap. For (ignoring entanglement) let the Schrödinger-picture state of a composite system of observer and environment at time $t$ be

$$
|\psi(t)\rangle \otimes\left(c|\chi(t)\rangle+c^{\prime}\left|\chi^{\prime}(t)\right\rangle\right)
$$

where $|\psi(t)\rangle$ is the state of the observer, and $c|\chi(t)\rangle+c^{\prime}\left|\chi^{\prime}(t)\right\rangle$ is the state of the environment, a superposition of macroscopically distinct states $|\chi(t)\rangle,\left|\chi^{\prime}(t)\right\rangle$. Perhaps the latter only differ with respect to macroscopic objects at enormously large spacelike distances - say, a radioactive decay that triggers a macroscopically significant event on a planetary system in the far side of Andromeda. In such a case, it is not at all obvious that the observer should be uncertain as to which branch, $|\psi(t)\rangle \otimes|\chi(t)\rangle$ or $|\psi(t)\rangle \otimes\left|\chi^{\prime}(t)\right\rangle$, she belongs. Her relative state in Everett's sense is the superposition $c|\chi(t)\rangle+c^{\prime}\left|\chi^{\prime}(t)\right\rangle$; in this there is no hint of uncertainty.

True enough, but for the mathematical identity:

$$
|\psi(t)\rangle \otimes\left(c|\chi(t)\rangle+c^{\prime}\left|\chi^{\prime}(t)\right\rangle\right)=c|\psi(t)\rangle \otimes|\chi(t)\rangle+c^{\prime}|\psi(t)\rangle \otimes\left|\chi^{\prime}(t)\right\rangle .
$$

We can read the relativization the other way: of the observer $|\psi(t)\rangle$ relative to the environment $|\chi(t)\rangle$ with amplitude $c$, and of another observer $|\psi(t)\rangle$ relative to the environment $\left|\chi^{\prime}(t)\right\rangle$ with amplitude $c^{\prime}$. It cannot be necessary to count the two observers as numerically the same, without further assumptions. Suppose, for example, they have their amplitudes as properties; if $c \neq c^{\prime}$ they are not even qualitatively the same.

Pursuit of the question of quantum non-locality leads on to relativity, where it connects with ordinary beliefs about probability in much the same way that the relativity of simultaneity connects with ordinary beliefs about tense (Saunders $[1995,1996])$. Here we shall stick to probability as it applies to events related by unambiguously timelike relations, as it figures in our practical lives.

What is needed is an atemporal perspective. That takes us to the quantum histories formalism: how does the distinction between overlap and divergence play out in the Heisenberg picture?

Schrödinger picture states at time $t$ are in $1-1$ relation to histories terminating at $t$, i.e. of the form $\alpha^{-}(t)$. We used a special case of this earlier (for single time histories). More generally, for a history $\alpha=\left\langle\alpha_{k_{n}}, \ldots, \alpha_{k_{1}}\right\rangle$ of configurations at times $t_{n}, . ., t_{1}$, let $C_{\alpha}$ be the time-ordered product of the associated Hiesenberg-picture projection operators (a chain operator; see appendix 1). The relationship between Schrödinger picture states at time $t_{n}$ resulting from the history $\alpha$ is, up to normalization:

$$
\left|\alpha^{-}(t)\right\rangle=\exp ^{-i H t / \hbar} C_{\alpha^{-}(t)}|\Omega\rangle=\exp ^{-i H t / \hbar} \sum_{\alpha \in \alpha^{-}(t)} C_{\alpha}|\Omega\rangle .
$$

That is, the Schrödinger picture state at time $t$ is (the forward evolution to $t$ ) of a superposition of Heisenberg-picture states $|\alpha\rangle=C_{\alpha}|\Omega\rangle$, all with the 


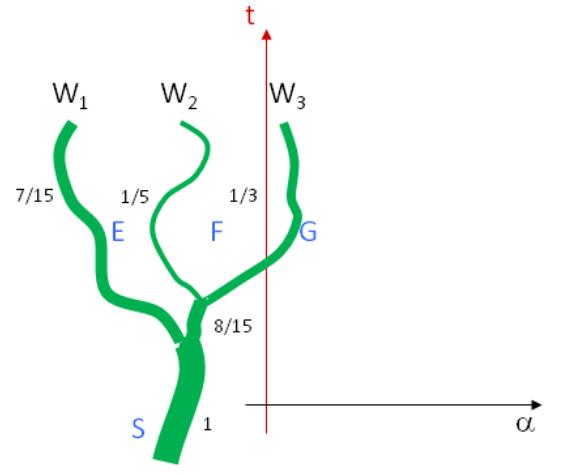

Branching

worlds

Fig. 1a

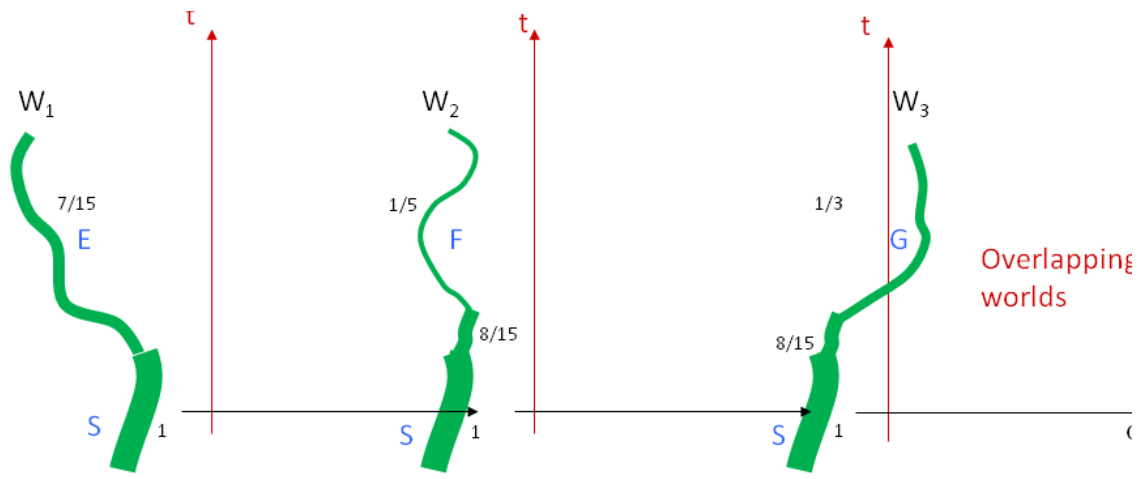

Fig. 1b

same sequence of configurations $\alpha^{-}(t)$ up to time $t$. Let 'worlds', as before, be represented by maximal branch vectors $|\alpha\rangle$. Do worlds overlap or diverge?

Here is the point made graphically. Fig 1a depicts the orbit of a Schrödinger picture wave-function that develops into a superposition of components $E, F$ and $G$, with squared norms as indicated; this explains how branches came by their name. But the branches themselves can be depicted in two ways, by either Fig 1b or Fig 1c. In Fig 1c it seems that branches do not overlap, whereas in Fig $1 \mathrm{~b}$ it seems that they do. Which of them is correct? Evidently the difference concerns only their amplitudes; of the two, Fig $1 \mathrm{c}$ is the obvious representation for Heisenberg picture vectors, which each have a unique amplitude. Fig 1b depicts rather graphs of Schrödinger picture states subject to state collapse, without renormalizing. Which of them is the 'correct' picture? But once stated in this way, the suspicion is that whether worlds in EQM diverge or overlap is underdetermined by the mathematics. One can use either picture; they are 


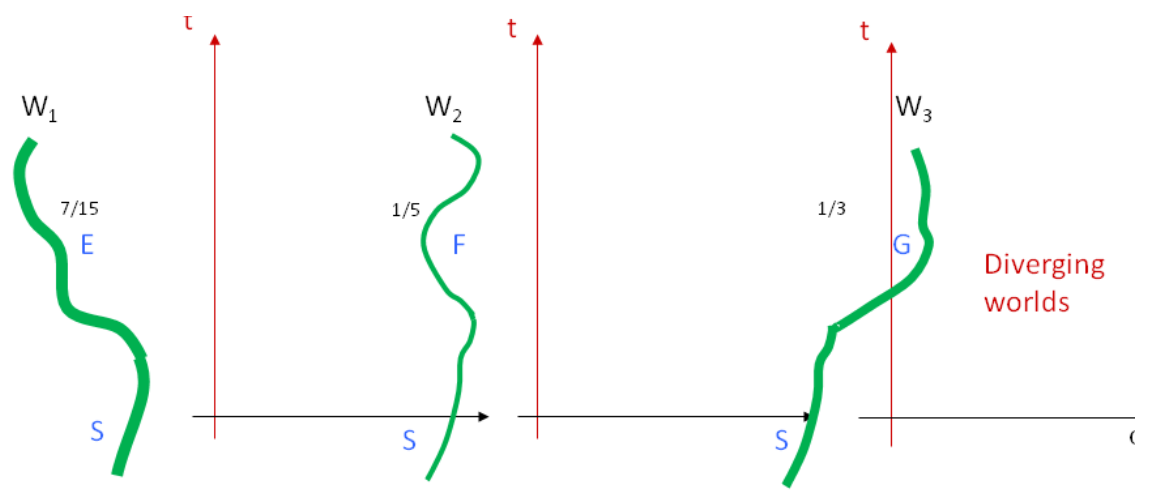

Fig. 1c

better or worse adapted to different purposes.

If so, it is pretty clear which is the right one for making sense of uncertainty. But of course Fig $1 \mathrm{c}$ is only a visual aid: can we be sure that the Heisenberg-picture branch vectors do not overlap? This, perhaps, is a technical philosophical question, to be settled by a theory of parts and wholes for vectors - by a vector mereology.

Alas, there is no such theory, or none on which there is any agreement. Mereology, if it is close to any branch of mathematics, is close to set theory (Lewis [1991)], but even there its links are controversial. We are going to have to make this up as we go along. And it is a good question, at this point, as to where the burden of proof really lies. Mereology has paid little or no dividends in pure mathematics, let alone in physics. It is at bottom an a priori metaphysical theory. A very good desideratum on any reasonable metaphysics is that it makes sense of our best physical theory, rather than nonsense.

Metaphysicians can, however, reasonably insist on an existence proof - a demonstration that at least one vector space mereology can be defined in terms of which maximal branches in EQM do not overlap. Here is a simple construction that depends essentially on the branching structure of a consistent history space. Let maximal branch vectors $|\alpha\rangle$ be defined as before. Any non-maximal branch vector $C_{\beta}|\Omega\rangle=|\beta\rangle$ can then be written (appendix 1):

$$
|\beta\rangle=\sum_{\alpha \in \beta}|\alpha\rangle .
$$

By the consistency condition, $\left\langle\alpha \mid \alpha^{\prime}\right\rangle=0$ for $\alpha \neq \alpha^{\prime}$; therefore for any two branch vectors $|\beta\rangle,|\gamma\rangle$ if $\langle\beta \mid \gamma\rangle \neq 0$ there exists a unique branch vector $|\delta\rangle$ and orthogonal branch vectors $\left|\beta^{\prime}\right\rangle,\left|\gamma^{\prime}\right\rangle$ such that

$$
|\beta\rangle=\left|\beta^{\prime}\right\rangle+|\delta\rangle,|\gamma\rangle=\left|\gamma^{\prime}\right\rangle+|\delta\rangle
$$


namely the vector:

$$
|\delta\rangle=\sum_{\alpha \in \beta \cap \gamma}|\alpha\rangle
$$

Our candidate mereology is then: a branch vector $|\epsilon\rangle$ is part of $\left|\epsilon^{\prime}\right\rangle$ ) (denote $P\left(|\epsilon\rangle,\left|\epsilon^{\prime}\right\rangle\right)$ if and only if either (a) $\epsilon=\epsilon^{\prime}$ or (b) there exists a non-zero branch vector $|\delta\rangle$ such that $\langle\epsilon \mid \delta\rangle=0,|\epsilon\rangle+|\delta\rangle=\left|\epsilon^{\prime}\right\rangle$.

Standard axioms of mereology are listed in appendix 2; it is easy to check that they are satisfied by the parthood relation just defined. From the overlap relation derived from $P$, we see that orthogonal vectors do not overlap.

It follows that every branch vector is part of the universal state $|\Omega\rangle$, as seems appropriate, but that orthogonal branch vectors have no parts in common. Since, by consistency, branching always produces orthogonal branch vectors, the branch vector representing Alice seeing spin down has no part in common with that representing Alice seeing spin up. On this basis branches of this kind diverge, in the philosophical sense, as do maximal branches.

But in what sense, it might be asked, is Alice represented as a part of a maximal branch vector $|\alpha\rangle$ ?- for the latter (on our candidate mereology) has no vectors as proper parts. The answer is that Alice is represented not by a vector at all, but by a sub-sequence $\beta$ of configurations of $\alpha=\left\langle\alpha_{+}, . ., \alpha_{-}\right\rangle$(see again $S 1$, and our use of ordered pairs $(\beta,|\alpha\rangle)$ - given which, questions about Alice and the part-whole relation in Alice's world are treated quasiclassically. It can hardly be objected that there must exist a uniform theory of mereology that applies across the board to emergent ontology. Far from it: that is a piece of metaphysics that has had little or no success in the special sciences. We concede only that one might be constructible in each domain.

With that it should be clear that our vector mereology on its own does not settle the question of uncertainty. Alice's successors downstream of branching diverge from each other, but they may still overlap with Alice prior to branching, even given our vector mereology. A branch can be coarse-grained from the time $t$ of branching in such a way that it is degenerate with respect to Alice seeing spin-up and Alice seeing spin-down. Thus a branch vector that represents only Alice's past $\alpha^{-}(t)$ at $t$ (of the form $\left|\alpha^{-}(t)\right\rangle=P_{\alpha_{k}}\left(t_{k}\right) \ldots P_{\alpha_{-}}\left(t_{-}\right)|\Omega\rangle, t_{k} \leq t$, the Heisenberg-picture analogue of Eq.(5)) represents Alice as containing all her possible future selves, that is, as overlapping completely, according to our mereology, with all her possible future selves, even if they do not overlap with each other.

And there, in a nutshell, is the contrary view: by all means take the parthood relation as specified, but let 'Alice' be indexed to time, and be represented by a Schrödinger picture vector accordingly (as given by Eq.(5)), or by the Heisenberg-picture branch vector $\left|\alpha^{-}(t)\right\rangle$, rather than by relativization to a maximal branch. Alice in this sense overlaps with all of her successors, and the grounds for self-locating uncertainty evaporate.

We always knew we had this option, however. This is to reject $S 1$ not on the grounds that worlds in EQM really overlap (on our proposed mereology they do not), but because superpositions of worlds overlap, and because (so goes the 
objection) persons and things should be represented by superpositions of worlds, superpositions of vectors.

But why should they? We can do the same in the case of diverging worlds of the sort usually considered by metaphysicians (having nothing to do with quantum mechanics), or as they arise in cosmic inflation (see e.g. Tegmark [2010]. In either case it is uncontroversial that worlds do not overlap, but still, sets of worlds do. Let a person or a thing up to time $t$ be a set of worlds, namely of those that contain that person or thing or one qualitatively identical up to time $t$ (which could be far in the future): then there is no uncertainty of where a person or thing is located among worlds that diverge after that time, either. But metaphysicians are unlikely to take this proposal very seriously, not least because it makes nonsense of much of what we say. Nor should we in the analogous case in EQM 18

To conclude: there is no good reason to think EQM is really a theory of overlapping worlds. If questions of overlap of branches are to be settled by appeal to the underlying mathematics, in terms of vector space structure, then there is at least one natural mereology in terms of which worlds that differ in some feature, since orthogonal, are non-overlapping. The semantics S1-S4 does not misrepresent the underlying mathematical structure of the theory; in terms of this mereology, it correctly describes worlds as non-overlapping.

But it does follow that the word 'branching' is something of a misnomer, in the context of EQM, in the philosophers' sense of the word. But then the word 'diverging' is also infelicitous. 'Branching' (inappropriately in EQM) suggests overlap, 'divergence' (inappropriately in EQM) suggests the absence of physical relations. I see nothing wrong with continuing to call EQM a theory of branching worlds, but only because the expression is well-established among physicist: ${ }^{19}$ and because it is fundamental to EQM that worlds, by superposition, do make up a dynamical unity - that they are all parts of the universal sate. At any rate, it will mark a new phase in the status of the Everett interpretation if the debate is over what the theory should be called.

Acknowledgements: My debt to David Wallace is obvious, but additional thanks are due to Harvey Brown, Hilary Greaves, and, especially, Alastair Wilson, for helpful comments and suggestions.

\footnotetext{
${ }^{18}$ Note added Sep 2016. Paul Tappenden has argued for the merits of this picture on other grounds (Tappenden [2011a]. He also offers arguments why, even if there is no self-locating uncertainty prior to branching in EQM, uncertainty can nevertheless be grounded on postbranching (Vaidman) uncertainty (Tappenden [2011b]). See also his criticisms of Saunders and Wallace [2008a,b] in Tappenden [2008]).

${ }^{19}$ Although some talk of 'parallel' worlds instead (see e.g. Tegmark [2010]). There is clearly a case for this terminology.
} 


\section{Appendix 1: Decoherent quantum histories}

For each $k$, let $P_{\alpha_{k}}, \alpha_{k}=1,2, \ldots$ be an exhaustive, commuting set of projection operators on a Hilbert space $\mathcal{H}$, (a partition of unity):

$$
\sum_{\alpha_{k}} P_{\alpha_{k}}=I, P_{\alpha_{k}} P_{\alpha_{k}^{\prime}}=\delta_{\alpha_{k} \alpha_{k}^{\prime}} P_{\alpha_{k}} .
$$

The $\alpha_{k}$ 's are to be thought of as coarse-grained cells of some parameter space (for the same of simplicity, say configuration space) ${ }^{20}$ Let $H$ be the Hamiltonian, assumeed time-independent; the associated Heisenberg picture operators for time $t_{k}$ are then:

$$
P_{\alpha_{k}}\left(t_{k}\right)=e^{i H t_{k} / \hbar} P_{\alpha} e^{-i H t_{k} / \hbar} .
$$

The simplest history spaces are those for which the partition of unity is the same at each time. Let $t_{1}<t_{2}<\ldots<t_{n}$, and consider a history $\alpha=\left\langle\alpha_{n}, \ldots, \alpha_{1}\right\rangle$; the associated chain (sometimes also called 'class') operator is:

$$
C_{\alpha}=P_{\alpha_{n}}\left(t_{n}\right) P_{\alpha_{n-1}}\left(t_{n-1}\right) \ldots P_{\alpha_{1}}\left(t_{1}\right) .
$$

The $C_{\alpha}$ 's are self-adjoint and positive (but not idempotent); they define a positive-operator-valued measure (POV measure). Acting on the state $\left|\Omega\left(t_{0}\right)\right\rangle$ at $t=0$ (abbreviate as $|\Omega\rangle$ ) we obtain branch state vectors $C_{\alpha}|\Omega\rangle=|\alpha\rangle$. These are in 1:1 correspondence with Schrödinger picture states $\left|\alpha\left(t_{n}\right)\right\rangle$, as would be obtained by measuring in sequence each $P_{\alpha_{k}}$ at time $t_{k}$, applying the projection postulate in each case. The correpondence (c.f. Eq.(5) section 4) is:

$$
\left|\alpha\left(t_{n}\right)\right\rangle=e^{-i H t_{n} / \hbar} C_{\alpha}|\Omega\rangle .
$$

The probability $p(\alpha$ of history $\alpha$ is the product of the probabilities for each individual step (where the probability of each step is as given by the Born rule). Formally:

$$
\left.p(\alpha)=\|\left|C_{\alpha}\right| \Omega\right\rangle \|^{2}=\operatorname{Tr}\left(C_{\alpha} \rho C_{\alpha}^{\dagger}\right)
$$

where $\rho=|\Omega\rangle\langle\Omega|$ is the density matrix for the state $|\Omega\rangle$ and ' $\operatorname{Tr}$ ' is the trace $\left(\operatorname{Tr}(O)=\sum_{k}\left\langle\phi_{k} \mid O \phi_{k}\right\rangle\right.$, for any operator $O$ and orthonormal basis $\left\{\left|\phi_{k}\right\rangle\right\}$ over $\mathcal{H}$ ). Likewise, the conditional probability of $\alpha$ (for $t_{n}<\ldots<t_{k+1}$ ) given $\beta$ (for $\left.t_{k}<\ldots<t_{1}\right)$ is

$$
p_{\rho}(\alpha / \beta)=\frac{\operatorname{Tr}\left(C_{\alpha * \beta} \rho C_{\alpha * \beta}^{\dagger}\right)}{\operatorname{Tr}\left(C_{\beta} \rho C_{\beta}^{\dagger}\right)} .
$$

where $\alpha * \beta$ is the history comprising $\beta$ (up to time $t_{k}$ ) and $\alpha$ (from $t_{k+1}$ to $t_{n}$ ). But whether or not these quantities can really be interpreted as probabilities - granted that repeatable measurements of the projectors $P_{\alpha_{k}}$ are not in fact performed - is another matter.

\footnotetext{
${ }^{20}$ Coarse-grainings of phase space are also possible, even yielding projective-valued measures; for example, by using von Neumann's construction (his 'building bricks of reality', cited by Everett [1973]; von Neumann[1932 p.409]. Jumping foreward, see Halliwell [2010] for the definition of decoherent histories in terms of coarse grainings (integrals) of local densities.
} 
Consider again the concept of coarse-graining of a parameter space (like configuration space). It extends naturally to chain operators: for each $k$, let $\left\{\overline{\alpha_{k}}\right\}$ be a coarse graining of $\left\{\alpha_{k}\right\}$, so that each finer-grained cell $\alpha_{k}$ is contained in some coarser grained cell $\overline{\alpha_{k}}$ in the parameter space. We can then speak of coarsening and fine-grainings of histories too. Now consider a set of histories with chain operators $\left\{C_{\alpha}\right\}$, and a coarse-graining with chain operators $\left\{C_{\bar{\alpha}}\right\}$. The two are simply related:

$$
C_{\bar{\alpha}}=\sum_{\alpha \in \bar{\alpha}} C_{\alpha}
$$

where the sum is over all finer-grained histories $\alpha$ contained within $\bar{\alpha}$. However, it by no means follows that the i probabilities of coarse-grainings of fine-grained histories are the sum of the probabilities of the fine-grained histories. The sum rule

$$
p(\bar{\alpha})=\sum_{\alpha \in \bar{\alpha}} p(\alpha) .
$$

is a substantive constraint 21 A sufficient condition for the sum rule is that vectors representing the fine-grained histories are approximately orthogonal:

$$
\left\langle C_{\alpha} \Omega \mid C_{\alpha^{\prime}} \Omega\right\rangle=\left\langle\alpha \mid \alpha^{\prime}\right\rangle \approx 0, \alpha \neq \alpha^{\prime} .
$$

Histories (for fixed Hamiltonian $H$ and state $\mid$ Omega $\rangle$ that satisfy (8) are called consistent (by Griffiths and Omnès); (medium) decoherent (by Gell-Mann and Hartle) ${ }^{22}$ Given consistency, Everett's relativization is a transitive relation even in time-like directions; it is automatically transitive in space-like directions by virtue of microcausality).

The additivity condition for a 1-place history, assuming (6), is automatically satisfied (Everett in his [1957] turned this reasoning around: assuming additivity, he derived (6)). It is satisfied by two-time histories as well, but in the general case it fails. A maximal set of consistent histories defines a consistent history space. If in addition the histories of such a space obey quasi-classical equations, the history space is called (following Gell-Man and Hartle [1993]) a quasiclassical domain.

It follows too that for any consistent history space there exists a fine-graining $\left\{P_{\alpha}\right\}$ which is consistent and for which, for any $t_{n}>t_{m}$ and for any $\alpha_{n}$ with $P_{\alpha_{n}}\left(t_{n}\right)|\Psi\rangle \neq 0$, there exists exactly one $\alpha_{m}$ such that

$$
P_{\alpha_{n}}\left(t_{n}\right) P_{\alpha_{m}}\left(t_{m}\right)|\Psi\rangle \neq 0
$$

(Griffiths [1993], Wallace [2012 p.92-94.]). That is, for each $\alpha_{n}$ at time $t_{n}$, there is a unique history preceding it - the set of histories can be fine-grained so as to have a purely branching structure (with no recombination of branches).

\footnotetext{
${ }^{21}$ For example, in the 2-slit experiment, it is not satisfied by histories so fine-grained as to show through which slit each particle goes.

${ }^{22}$ The necessary and sufficient condition for the sum rule (7) is that only the real part of (9) vanish (or approximately vanish). But the stronger condition is the more robust, and is automatically satisfied in a quasiclassical domain.
} 
The consistency condition and the quantum histories formalism is widely considered a generalization of quantum theory as, fundamentally, a theory of probability. As such there is a continuum infinity of consistent history spaces available - new resources for the exploration of quantum systems, indeed. But from the point of view of EQM, consistency is far too weak a condition to give substance to the notion of histories as autonomous and robust structures in the universal sate, our abiding criteria for the existence of worlds.

\section{Appendix 2: Axioms of mereology}

We write 'Pxy' for ' $x$ is part of $y$ '; this relation is reflexive, transitive, and antisymmetric. Defined notions are:

Overlap $O x y \underset{d e f}{=} \exists z(P z x \& P z y)$.

Underlap $U x y \underset{\text { def }}{=} \exists z(P x z \& P y z)$.

Fusion $x \sqcup y \underset{d e f}{=} \iota z \forall w(P x w \& P y w \rightarrow P z w)$.

(In words, $x$ and $y$ overlap if a part of $x$ is a part of $y$; they underlap if there is something of which they are both parts; and their fusion is the unique thing that is a part of anything of which $x$ and $y$ are both parts.) The elementary axioms of mereology are:

M1 $\forall x(x \sqcup x)=x$

M2 $\forall x \forall y(U x y \rightarrow \exists z(z=x \sqcup y))$

M3 $\forall z(P x z \& P y z \rightarrow P x \sqcup y z)$

(the fusion of anything with itself is itself; if two things underlap then their fusion exists; anything which underlaps two things is part of the fusion of those things). Define the parthood relation as above:

Vector Part $P(|\beta\rangle,|\gamma\rangle)$ if and only if either (a) $\beta=\gamma$, or (b) there exists a non-zero branch $|\delta\rangle$ such that $\langle\delta \mid \beta\rangle=0,|\beta\rangle+|\delta\rangle=|\gamma\rangle$.

It is simple to check that $P$ is reflexive, transitive, and antisymmetric. The fusion operation as defined by $P$ is:

- If $\langle\beta \mid \gamma\rangle=0,|\beta\rangle \sqcup|\gamma\rangle=|\beta\rangle+|\gamma\rangle$

- If $\langle\beta \mid \gamma\rangle \neq 0, \beta \in \gamma,|\beta\rangle \sqcup|\gamma\rangle=|\gamma\rangle ; \gamma \in \beta,|\beta\rangle \sqcup|\gamma\rangle=|\beta\rangle$.

It clearly satisfies $M 1-M 3$. 


\section{References}

Aharonov, Y., D. Albert, and L. Vaidman [1988], 'How the result of a measurement of a component of the spin of a spin- $\frac{1}{2}$ particle can turn out to be 100', Physical Review Letters 60, 1351-54.

Aharanov, Y., and L. Vaidman [1993], 'Protective measurements', Physics Letters A 178 p. 38.

Davidson, D. [2001], Inquiries into Truth and Interpretation (2nd ed.). Oxford University Press.

Deutsch, D. [1999], 'Quantum theory of probability and decisions', Proceedings of the Royal Society of London A455, 3129-3137. Available online at/http://arxiv.org/abs/quantph/9906015.

— [2010], 'Apart from universes', in Saunders et al [2010], pp.542-52.

Everett III, H. [1957], 'Relative state formulation of quantum mechanics', Reviews of Modern Physics 29, 454-62.

- [1973], 'Theory of the universal wave-function', in The Many-Worlds Interpretation of Quantum Mechanics, B. De Witt and N. Graham (eds.), pp.3-140, Princeton University Press.

Gell-Mann, M. and J.B. Hartle [1990], 'Quantum Mechanics in the light of quantum cosmology', in Complexity, Entropy, and the Physics of Information, W.H. Zurek, ed., Reading, Addison-Wesley.

— [1993], 'Classical equations for quantum systems', Physical Review D 47, 3345382. Available online at http://arxiv.org/abs/gr-qc/9210010.

Greaves, H. [2004], 'Understanding Deutsch's probability in a deterministic multiverse', Studies in History and Philosophy of Modern Physics 35, 423-56. Available online at http://philsci-archive.pitt.edu/archive/00001742/

— [2007], 'On the Everettian epistemic problem', Studies in History and Philosophy of Modern Physics 38, 120-152. Available online at http://philsci-archive.pitt.edu/archive/00002953

Greaves, H. and W. Myrvold [2010], 'Everett and evidence', in Saunders et al [2010], pp.264-304.

Griffiths, R. [1993], 'Consistent interpretation of quantum mechanics using quantum trajectories', Physical Review Letters 70, 2201-2204.

Halliwell, J. [2010], 'Macroscopic superposition, decoherent histories, and the emergence of hydrodynamic behaviour', in Saunders et al [2010], pp.99-117.

Ismael, J. [2003], 'How to combine chance and determinism: Thinking about the future in an Everett universe', Philosophy of Science 70, 776-790.

Johnston, M. [1989], 'Fission and the facts', Philosophical Perspectives, 3, 369-397.

Kent, A. [2010], 'One world versus many: the inadequacy of Everettian accounts of evolution, probability, and scientific confirmation', in Saunders et al [2010].

Ladyman, J. and D. Ross [2007], Every Thing Must Go: Metaphysics Naturalized, Oxford.

Lewis, D. [1973], Counterfactuals. Harvard.

- [1986a], Philosophical Papers, Vol. 2, Oxford: Oxford.

- [1986b], On the Plurality of Worlds, Blackwell.

- [1991], Parts of Classes, Blackwell. 
Lubkin, E. [1979], 'An application of ideal experiments to quantum mechanical measurement theory', International Journal of Theoretical Physics 18, 165-77.

Papineau, D. [1996], 'Comment on Lockwood', British Journal for the Philosophy of Science 47, 233-41.

Saunders, S. [1994], 'Decoherence and evolutionary adaptation', Physics Letters A 184, p.1-5.

- [1997], 'Naturalizing metaphysics', The Monist 80, 44-69.

- [1998], 'Time, quantum mechanics, and probability', Synthese, 114, pp.405-44. Available online at http://arxiv.org/abs/quant-ph/0112081.

— [2004], 'Derivation of the Born Rule from Operational Assumptions', Proceedings of the Royal Society A 460, 1-18. Available online at https://arxiv.org/abs/quant$\mathrm{ph} / 0211138$

— [2005], 'What is probability?', in Quo Vadis Quantum Mechanics, A. Elitzur, S. Dolev, and N. Kolenda, eds., Springer. Available online at http://arxiv.org/abs/quantph/0412194.

Saunders, S., and D. Wallace [2008a] 'Branching and uncertainty', British Journal for the Philosophy of Science 59, 293 - 305. Available online at http://philsciarchive.pitt.edu/archive/00003811/.

— [2008b], 'Saunders and Wallace reply', British Journal for the Philosophy of Science 59, 315-17.

Saunders, S., J. Barrett, A. Kent, and D. Wallace (eds.)[2010], Many Worlds? Everett, Quantum Theory, and Reality, Oxford.

Savage, L. [1954], The Foundations of Statistics, Wiley.

Tappenden, P. [2008], 'Comment on Saunders and Wallace', British Journal for the Philosophy of Science 59, 306-314.

— [2011a], 'A metaphysics for semantic internalism', Metaphysica12, 125-36. Available online at http://philsci-archive.pitt.edu/8721/.

- [2011b], Evidence and uncertainty in Everetts multiverse, British Journal for the Philosophy of Science 62, pp. 99123. Available online at http://philsciarchive.pitt.edu/5046/.

Tegmark, M. [2010], 'Many worlds in context', in Saunders et al [2010] pp.553-81.

Uffink, J. [2000] 'How to protect the interpretation of the wave function against protective measurements'. Available online at http://arxiv.org/abs/quant-ph/9903007v1.

Vaidman, L. [1998], 'On schizophrenic experiences of the neutron or why we should believe in the many-worlds interpretation of quantum theory', International Studies in the Philosophy of Science 12, 245-261.

Van Fraassen, B. [1980], The Scientific Image, Oxford: Clarendon Press.

Von Neumann, J. [1932], Mathematische Grundlagen Der Quantenmechanik, translated by R.T. Beyer as Mathematical Foundations of Quantum Mechanics, Princeton University Press, 1955.

Wallace, D. [2005], 'Language use in a branching universe', available online at http://philsci-archive.pitt.edu/archive/00002554.

- [2006], 'Epistemology quantized: circumstances in which we should come to believe in the Everett interpretation', British Journal for the Philosophy of Science 57, 655-689. Available online at http://philsci-archive.pitt.edu/archive/00002839. 
— [2010], 'How to prove the Born rule', in Saunders et al [2010] pp.227-63. Available online at http://philsci-archive.pitt.edu/4709/

- [2012], The Emergent Multiverse: Quantum Theory according to the Everett Interpretation, Oxford.

Wilson, A. [2011], 'Macroscopic ontology in Everettian quantum mechanics, Philosophical Quarterly 61, pp. 36382.

— [2013], 'Objective probability in Everettian quantum mechanics', Britisch Journal for the Philosophy of Science 64, 709-37.

Zurek, W. [2005], 'Probabilties from entanglement, Born's rule $p_{k}=\left|\psi_{k}\right|^{2}$ from envariance', Physical Review A71, 052105. Available online at https://arxiv.org/abs/quant$\mathrm{ph} / 0405161$ 